\title{
Vital Signs Time Point
}

National Cancer Institute

\section{Source}

National Cancer Institute. Vital Signs Time Point. NCI Thesaurus. Code C83159.

A point in time that the vital signs assessment takes place. 